\title{
Throughput Evaluation of Downlink Multiuser-MIMO OFDM-LTE System
}

\author{
Patteti Krishna', Tipparti Anil Kumar', Kalithkar Kishan Rao ${ }^{3}$ \\ ${ }^{1}$ JNT University, Hyderabad, Telangana, India \\ ${ }^{2}$ Department of ECE, SR Engineering College, Warangal, Telangana, India \\ ${ }^{3}$ Vaagdevi College of Engineering, Warangal, Telangana, India \\ Email: kpatteti@gmail.com, tvakumar2000@yahoo.co.in, kishanrao6@gmail.com
}

Received March 2015

\begin{abstract}
Recently, the mobile communication industry is moving rapidly towards long-term evolution (LTE) systems. LTE aims to provide improved service quality over 3G systems in terms of throughput, spectral efficiency, latency, and peak data rate, and MIMO technique is one of the key enablers of the LTE system for achieving these diverse goals. Among several operational modes of MIMO, multiuser MIMO (MU-MIMO) in which the base station transmits multiple streams to multiple users, has received much attention as a way for achieving improvement in performance. In this paper we present a Multiuser MIMO-OFDM-based simulator that includes the main physical layer functionalities and calculate the throughput of LTE Frequency Division Duplex (FDD) and Time Division Duplex (TDD) systems. The simulator has been used to evaluate the performance of the 3GPP Long-Term Evolution (LTE) technology.
\end{abstract}

\section{Keywords}

3GPP LTE, OFDM, MIMO, MU-MIMO, FDD, TDD and Throughput

\section{Introduction}

LTE is one of the most promising wirelesses-technology platforms for the future. The version being deployed today is just the beginning of a series of innovations that will increase performance, efficiency, and capabilities. To address the growing mobile broadband demand, the 3 GPP standards body released the next technological step, Long Term Evolution (LTE) [1] [2]. LTE is designed to substantially improve end-user throughputs, increase sector capacity and reduce user plane latency. Among many features in the LTE which supports up to 3Gbps throughput in downlink, Multi user multiple-input-multiple-output (MU-MIMO) scheme has been identified as one of the key enablers for achieving a high spectral efficiency. Both in theory and design perspectives, MU-MIMO systems have several unique features distinct from single user MIMO (SU-MIMO) systems [3]. To make up for the shortcomings of SU-MIMO, early LTE [4] standards (Rel. 8 and 9) defined a primitive form of the MU-MIMO mode. Many of us might have heard about LTE peak throughput is $300 \mathrm{Mbps}$, but how many of us know how we calculate that? This paper provides the information, how this number is calculated? In this pa- 
per, we explained the calculations of theoretical throughput for both the LTE Frequency Division Duplex (FDD) and Time Division Duplex (TDD) systems [5] [6].

\section{System Model}

We consider a downlink MIMO-OFDM system with $m$ users, $N_{f}$ subcarriers, $n_{T}$ transmit antennas at the base station, and $n_{R m}$ receive antennas at the $m^{\text {th }}$ mobile station. The data for a particular user, for example, $m$, are transmitted in packets, and denoted as $\mathbf{S}_{m}[n] \in \mathrm{C}^{N_{m}}$ for $n=1, \cdots, N_{f}$, where $N_{m}$ is the number of spatial sub channels that are offered from the multiple transmit antennas. Since the channels are assumed to be quasi-static fading from one OFDM symbol to another, the time index is omitted for simplicity. We also assume that the elements in $\mathbf{S}_{m}[n]$ are independent identically distributed (i.i.d.) random variables. Let $p_{m}[k, n]$ denotes the power that is allocated to the $k$ th spatial sub channel on the $n^{\text {th }}$ subcarrier of user $m$.

With proper guard timing and cyclic prefix, the estimated frequency domain signal is

$$
\widehat{\mathbf{S}_{m}}[n]=\mathbf{R}_{m}[n]\left(\mathbf{H}_{m}[n] \sum_{m=1}^{M} \mathbf{T}_{m}[n]\right) \widehat{\mathbf{S}_{m}}[n]+\boldsymbol{\eta}_{m}[n]
$$

where $\mathbf{H}_{m}[n]$ denotes the MIMO channel matrix from the base station to user $m$ at subcarrier $n$. The data symbol vector $\mathbf{S}_{m}[n]$ is post multiplied by the transmit beam forming matrix $\mathbf{T}_{m}[n] \in \mathrm{C}^{K} \times N^{m}$ before transmitting from the antennas. Having set the transmit power to be $p_{m}[k, n]$, the columns of $\mathbf{T}_{m}[n]$ are normalized to 1 . The same holds for the receive beam forming matrix $\mathbf{R}_{m}[n]$. In addition, the noise $\boldsymbol{\eta}_{m}[n]$ is assumed to be i.i.d. complex Gaussian with zero mean and variance of $\sigma^{2}$. The fidelity of the signal $\widehat{\mathbf{S}_{m}}[k, n]$ is measured by its SINR, which is given by

$$
\Gamma_{m}[k, n]=\frac{p_{m}[k, n] \mathbf{R}_{m}[k, n] \mathbf{H}_{m}[k, n] \mathbf{T}_{m}[k, n]^{2}}{\sum_{m=1}^{M} \sum_{k=1}^{N_{m}} p_{m}[k, n] \mathbf{R}_{m}[k, n] \mathbf{H}_{m}[k, n] \mathbf{T}_{m}[k, n]^{2}+\sigma^{2}}
$$

With the assumption that the interference terms in (2) are Gaussian and independent, from the information-theoretic viewpoint, the achievable aggregate rate for user $m$, which is denoted as $v_{m}$, becomes

$$
\sum_{n=1}^{N_{f}} \sum_{k=1}^{N_{m}} \log _{2}(1+\Gamma[k, n])
$$

Therefore, the system throughput is

$$
v=\sum_{m=1}^{M} v_{m}
$$

\section{Maximum Throughput with Maximum Bandwidth}

For any system throughput is calculated as symbols per second. Further it is converted into bits per second depending on the how many bits a symbol can carry. In LTE for $20 \mathrm{MHz}$, there are 100 Resource Blocks and each Resource block have $12 \times 7 \times 2=168$ Symbols per ms in case of Normal CP. So there are 16,800 Symbols per ms or 16,800,000 Symbols per second or 16.8 Msps. If modulation used is 64 QAM (6 bits per symbol) then throughput will be $16.8 \times 6=100.8 \mathrm{Mbps}$ for a single chain.

For a LTE [7] system with $4 \times 4$ MIMO (4T4R) the throughput will be four times of single chain throughput. i.e. 403.2 Mbps. Many simulations and studies show that there is $25 \%$ of overhead used for Controlling and signalling. So the effective throughput will be $300 \mathrm{Mbps}$. The $300 \mathrm{Mbps}$ number is for downlink and not valid for uplink. In uplink we have only one transmit chain at UE end. So with $20 \mathrm{MHz}$ we can get Maximum of 100.8 Mbps as calculation shown above. After considering 25\% of overhead we get 75 Mbps in uplink. This is the way how we get the number of throughput $300 \mathrm{Mbps}$ for Downlink and 75 Mbps for Uplink shown everywhere.

\section{Duplex Schemes}

Spectrum flexibility is one of the key features of LTE. In addition to the flexibility in transmission bandwidth, LTE also supports operation in both paired and unpaired spectrum by supporting both FDD-and TDD-based 
duplex operation with the time frequency structures. Although the time-domain structure is, in most respects, the same for FDD and TDD, there are some differences, most notably the presence of a special sub frame in the case of TDD. The special sub frame is used to provide the necessary guard time for downlink-uplink switching shown in Table 1.

\section{DL and UL Throughput Calculation for LTE FDD}

The FDD system has a paired spectrum, same bandwidth for Downlink as well as for Uplink. $20 \mathrm{MHz}$ FDD system have $20 \mathrm{MHz}$ for Downlink and $20 \mathrm{MHz}$ for Uplink. For throughput calculation, suppose:

Bandwidth-20 MHz

Multiplexing scheme-FDD

UE category-Cat. 3

Modulation supported—as per Cat 3 TBS index 26 for DL (75376 for 100RBs) and 21 for UL (51024 for 100 RBs)

So the throughput can be calculated by a simple formula:

Throughput $=$ Number of Chains $\times$ TB size

So DL throughput $=2 \times 75376=150.752 \mathrm{Mbps}$

UL throughput $=1 \times 51024=51.024$ Mbps

As we have 2 receive chains and one transmits chain.

\section{LTE TDD and Its Frame Structure}

Before starting throughput calculation, let's become familiar with LTE-TDD [6]. As stated earlier, TDD is unpaired spectrum. We have to use same bandwidth for DL and UL on time sharing basis. Suppose if we have 20 $\mathrm{MHz}$ spectrum, we have to use this $20 \mathrm{MHz}$ bandwidth for both DL and UL.LTE TDD frame structure is shown in Figure 1. The TD frame consists of Downlink sub frame, Uplink and Special sub frame.

There are seven possible configurations for LTE TDD frame as shown below. Here D-is downlink, S-for Special sub frame and U- for Uplink. As shown $5 \mathrm{~ms}$ periodicity frame have two "S" sub frame and $10 \mathrm{~ms}$ frames have only one "S" sub frame.

Special sub frame has 9 different configurations [8]. A special sub frame is divided into Downlink Pilot Time Slot (DwPTS), Guard Period (GP) and Uplink Pilot Time Slot (UpPTS) depending upon the number of symbols.

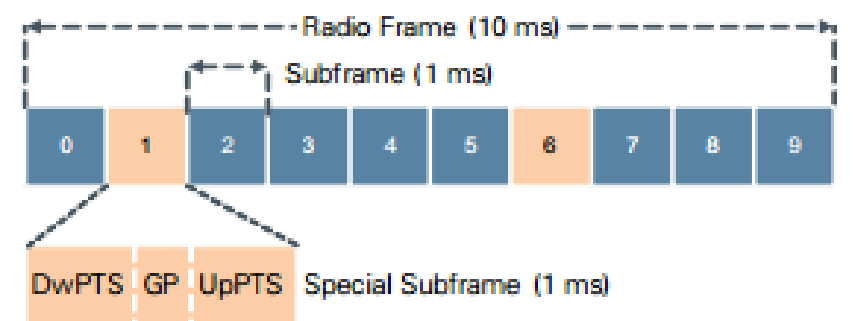

Figure 1. TDD frame structure.

Table 1. Uplink-downlink allocations.

\begin{tabular}{ccccccccccccc}
\hline UL-DL configuration & \multicolumn{1}{c}{ Downlink to up link switch periodicity } & \multicolumn{1}{c}{ Sub frame number } \\
\hline & & 0 & 1 & 2 & 3 & 4 & 5 & 6 & 7 & 8 & 9 \\
\hline 0 & $5 \mathrm{~ms}$ & $\mathrm{D}$ & $\mathrm{S}$ & $\mathrm{U}$ & $\mathrm{U}$ & $\mathrm{U}$ & $\mathrm{D}$ & $\mathrm{S}$ & $\mathrm{U}$ & $\mathrm{U}$ & $\mathrm{U}$ \\
1 & $5 \mathrm{~ms}$ & $\mathrm{D}$ & $\mathrm{S}$ & $\mathrm{U}$ & $\mathrm{U}$ & $\mathrm{D}$ & $\mathrm{D}$ & $\mathrm{S}$ & $\mathrm{U}$ & $\mathrm{U}$ & $\mathrm{D}$ \\
2 & $5 \mathrm{~ms}$ & $\mathrm{D}$ & $\mathrm{S}$ & $\mathrm{U}$ & $\mathrm{D}$ & $\mathrm{D}$ & $\mathrm{D}$ & $\mathrm{S}$ & $\mathrm{U}$ & $\mathrm{D}$ & $\mathrm{D}$ \\
3 & $10 \mathrm{~ms}$ & $\mathrm{D}$ & $\mathrm{S}$ & $\mathrm{U}$ & $\mathrm{U}$ & $\mathrm{U}$ & $\mathrm{D}$ & $\mathrm{D}$ & $\mathrm{D}$ & $\mathrm{D}$ & $\mathrm{D}$ \\
4 & $10 \mathrm{~ms}$ & $\mathrm{D}$ & $\mathrm{S}$ & $\mathrm{U}$ & $\mathrm{U}$ & $\mathrm{D}$ & $\mathrm{D}$ & $\mathrm{D}$ & $\mathrm{D}$ & $\mathrm{D}$ & $\mathrm{D}$ \\
5 & $10 \mathrm{~ms}$ & $\mathrm{D}$ & $\mathrm{S}$ & $\mathrm{U}$ & $\mathrm{D}$ & $\mathrm{D}$ & $\mathrm{D}$ & $\mathrm{D}$ & $\mathrm{D}$ & $\mathrm{D}$ & $\mathrm{D}$ \\
6 & $5 \mathrm{~ms}$ & $\mathrm{D}$ & $\mathrm{S}$ & $\mathrm{U}$ & $\mathrm{U}$ & $\mathrm{U}$ & $\mathrm{D}$ & $\mathrm{S}$ & $\mathrm{U}$ & $\mathrm{U}$ & $\mathrm{D}$ \\
\hline
\end{tabular}




\section{DL and UL Throughput Calculations for LTE TDD}

TDD system throughput calculations are somewhat complex as compared to FDD system as same spectrum is used by uplink, downlink and for the guard period (Used for transition from downlink to uplink) [9].

For throughput calculation, suppose:

Bandwidth-20 MHz

Multiplexing Scheme-TDD

TDD Configuration-2 (D-6, S-2 and U-2)

Special Sub frame configuration-7 (DwPTS-10, GP-2 and UpPTS-2)

UE category-Cat. 3

Modulation supported-as per Cat 3 TBS index 26 for DL (75376 for 100 RBs) and 21 for UL (51024 for 100

RBs). Throughput in TDD can be calculated by following formula

DL Throughput $=$ Number of Chains $\times$ TB size $\times($ Contribution by DL Sub frame + Contribution by DwPTS in SSF).

UL Throughput $=$ Number of Chains $\times$ TB size $\times$ (Contribution by UL Sub frame + Contribution by UpPTS in SSF). TB size for DL is 75376 and for UL it is 51,024 for category 3 UE.

Let's calculate throughput for the above assumptions:

DL throughput $=2 \times 75376 \times[(0.6+0.2 \times(10 / 14)]$

Here $0.6 \%$ or $60 \%$ contribution is by 6 DL sub frame and [0.2(10/14)] factor contribution by Special sub frame comes twice whose 10 symbols out of 14 are for downlink.

So DL throughput $=2 \times 75376 \times(0.742857)=111.9872 \mathrm{Mbps}-112 \mathrm{Mbps}$.

In same manner UL throughput will be

UL throughput $=1 \times 51024 \times[(0.2+0.2 \times(2 / 14)]$

Here $0.2 \%$ or $20 \%$ contribution is by 2 UL sub frame and $[0.2 \times(2 / 14)]$ factor contribution by Special sub frame comes twice whose 2 symbols out of 14 are for uplink.

So UL throughput $=1 \times 51024 \times(0.228571)=11.66263-12 \mathrm{Mbps}$.

\section{Conclusion}

In this paper, we discussed about LTE system throughput calculation for both TDD and FDD system. 3 GPP LTE technology support both TDD and FDD multiplexing. The paper describes all the factors which affect the throughput like Bandwidth, Modulation, UE category and multiplexing. It also describes how we get throughput $300 \mathrm{Mbps}$ in DL and $75 \mathrm{Mbps}$ in UL and what are assumptions taken to calculate the same. Paper describes the steps and formulae to calculate the throughput for FDD system for TDD Configuration 1 and Configuration 2.

\section{References}

[1] Dahlman, E., Parkvall, S. and Skold, J. (2011) 4G LTE/LTE-Advanced for Mobile Broadband. Academic Press.

[2] Sesia, S., Toufik, I. and Baker, M. Lte-The Umts Long Term Evolution from Theory to Practice. 2nd Edition.

[3] Boccardi, F., Clerckx, B., Ghosh, A., Hardouin, E., Jongren, G., Kusume, K., Onggosanusi, E. and Tang, Y. (2012) Multiple-Antenna Techniques in LTE-Advanced. IEEE Communications Magazine, 50, 114-121

[4] 3GPP TS 36.213. Evolved Universal Terrestrial Radio Access (E-UTRA) Physical Layer Procedures.

[5] (2009) 3GPP LTE for TDD Spectrum in the Americas.

[6] Ratasuk, R., et al. (2008) TDD Design for UMTS Long Term Evolution. PIMRC-2008, Cannes.

[7] Holma, H. and Toskala, A. (2009) LTE for UMTS: OFDMA and SC-FDMA Based Radio Access, 267.

[8] (2013) Cisco Visual Networking Index: Global Mobile Data Traffic Forecast Update, 2012-2017.

[9] Toward 4G. http://www.nttdocomo.com/technologies/future/toward/index.html 